small, and it took about 70 clinic-years to assemble the 800 patients studied. Moreover, the results from all the centres are not consistent. While two centres, Cincinatti and Minneapolis, contributed less than a quarter of all the patients they provided no fewer than half the deaths in the placebo and tolbutamide groups. The accuracy of some of the causes of death given must be in doubt, since necropsies were done in only one-third of the cases and in different proportions in the two groups (half in the tolbutamide group, one-quarter in the placebo group). More of the deaths in the tolbutamide group occurred in hospital $(70 \%)$ than in the placebo group (52\%). On the other hand, of 205 middle-aged diabetics in the placebo group observed for up to eight and three-quarter years not one is recorded as having died from myocardial infarction.

Another unusual feature of this study, in which as many as $72 \%$ of the patients were women, was that the excess mortality from cardiovascular disease among the tolbutamide group was almost confined to women. Among the men there were 7 deaths in the placebo group as against 11 in the tolbutamide group, compared with 3 and 15, respectively, in the women. Again, of all the patients in the study half were non-white (a much higher proportion than in the general population); and the excess of female cardiovascular deaths was mainly among white women. Hence in so far as this study suggests an increased risk of cardiovascular death among diabetics treated with tolbutamide, this risk is mainly for white women.

These results are at variance with those reported by $\mathrm{H}$. Keen and R. J. Jarrett in Great Britain ${ }^{2}$ and by J. Paasikivi in Sweden. ${ }^{3}$ Neither of these studies shows an increased risk of cardiovascular disease in the groups of patients treated with tolbutamide. In fact, they suggest the reverse.

If the results are in some ways open to question, so too is the way they have been handled. It is desirable that the results of drug trials, as of other medical research, should be assessed in a cool and critical fashion. This becomes difficult when they reach the lay press before doctors as a whole have had the chance to consider them, as has happened in the present case. ${ }^{4}$ Why have the conclusions drawn from this report been so urgent, certain, and final? The tolbutamide study has now been shut down. The trial is in many ways thorough and excellent, but several criticisms can be made and the F.D.A. itself admits that it contains "a number of limitations." In June the American Diabetes Association issued a statement saying that the evidence did "not appear to warrant abandoning the present accepted methods of treatment of diabetes-diet, diet with oral agents, or diet and insulin as indicated." Yet now, with no new evidence available, the Ar.terican Diabetes Association has associated itself with the F.D.A. view that oral drugs should be used only when diet alone is ineffective and insulin is impracticable or unacceptable. This advice is the more surprising since the co-operative study showed that less than half the patients on a variable dose of insulin actually adhered strictly to their treatment. Moreover, insulin treatment itself has drawbacks, including hypoglycaemia and weight gain, with the possible risks resulting from them.

Since the co-operative study has not yet been published in its final form the medical profession in general, whether in the U.S.A. or elsewhere, has had no opportunity to evaluate it. That it needs unusually careful scrutiny is certain, for some aspects of it are open to question. In Britain it would be wise to defer judgement on what action to take uncil the report has been published and studied rather than to follow blindly the F.D.A.'s lead. The calm attitude throughout of the British Diabetic Association, which has also advised caution, is to be welcomed.

\section{The Immunoglobulins}

When A. Tiselius ${ }^{1}$ in 1937 first separated the plasma proteins by their different mobilities in an electric field, he distinguished three varieties of globulin designated $\alpha, \beta$, and $\gamma$ in decreasing order of migration. It soon became apparent that the antibody property of an immune serum - that is, its capacity to react specifically in a detectable fashion with some foreign substance-resided in the $\gamma$ globulin fraction, and the term "immunoglobulin" is now applied to any constituent of this fraction that can be shown to possess either specific antibody properties or the structure now known to be characteristic of them.

By a combination of physical, chemical, and immunological methods the human immunoglobulins have been divided into five classes designated $\mathrm{G}, \mathrm{A}, \mathrm{M}, \mathrm{D}$, and $\mathrm{E}$. In normal human serum $\mathrm{G}$ is the most abundant and $\mathrm{E}$ the least. All five classes are constructed on the same basic plan-namely, four peptide chains, two short and two long, the former each composed of about 214 amino-acids, the latter of about twice that number. They are now generally referred to as the light (L) and heavy $(\mathrm{H})$ chains with respective molecular weights of about 25,000 and 50,000 , the complete molecule thus possessing a molecule weight of 150,000 . Whereas most of the molecules of immunoglobulin $\mathrm{G}$ (IgG) exist in this form, the IgM molecule is made up of five such units covalently linked to each other by sulphur bonds between cysteine residues. By careful chemical reduction of these bonds the IgM molecule can be dissociated into its constituent units of about 150,000 molecular weight. IgD and $\operatorname{IgE}$ probably exist mainly in the monomer form (single units) like IgG, but IgA shows a considerable tendency to aggregate into dimers and larger polymers.

The difference between the five classes of immunoglobulin lies exclusively in the heavy chains. The light chains, of which two forms exist, are common to all the immunoglobulins, but each class, G, A, M, D, and E, has its own characteristic heavy chain designated $\gamma, \alpha, \mu, \delta$, and $\varepsilon$ respectively. These, being immunologically distinct, can be readily detected and differentiated by specific antibodies.

The mutual relationship of the four constituent chains in the basic immunoglobulin molecule has been largely established by the study of the fragments released by proteolytic enzymes. Thus R. R. Porter ${ }^{2}$ showed that rabbit immunoglobulin digested with papain yielded two distinct types of fragment. Only one of them retained the antibody specificity of the parent molecule and was therefore labelled Fab (i.e., antibody containing fragment), while the other, the Fc fraction, had no such biological activity but could be readily crystallized, indicating a degree of homogeneity not present in the Fab fraction. The Fab fragments, though retaining antibody specificity, were incapable of precipitating antigen, because each fragment retained only one of the two combining sites of the parent molecule. But A. Nisonoff and colleagues ${ }^{3}$ showed that if pepsin was used in place of papain the homologous fragment, now termed $F\left(a b^{\prime}\right)_{2}$, still retained the capacity of immunologically specific precipitation and was thus still bivalent. The results of these and similar experiments led to the Porter diagram ${ }^{4}$ of immunoglobulin structure, which also shows the makeup of the various fragments of proteolytic digestion (see Fig.).

Although the $\mathrm{Fc}$ portion of the $\mathrm{H}$ chains plays no part in the immunological specificity of the various immunoglobulins, it is of considerable biological significance, because the properties of complement fixation, ability to pass the 
$F\left(a b^{\prime}\right)_{2}$

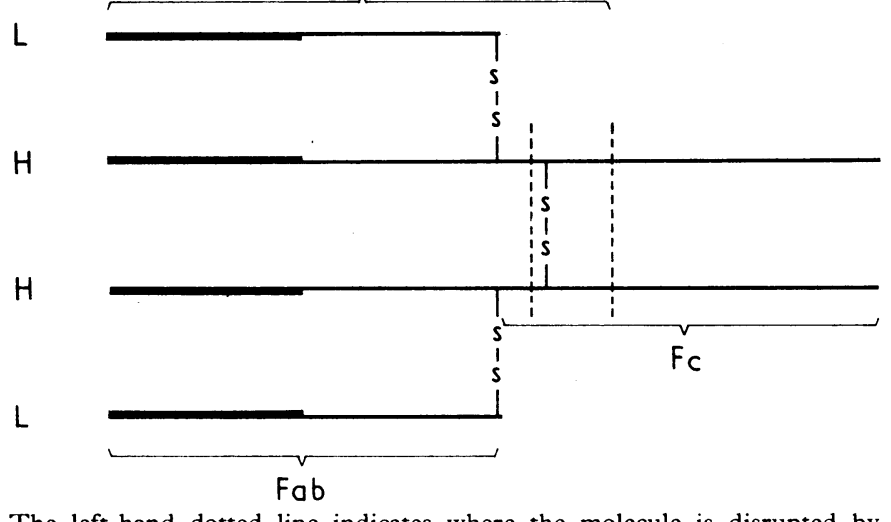

The left-hand dotted line indicates where the molecule is disrupted by papain the right-hand line the site of pepsin disruption. The heavily marked lines indicate the areas where the specific combining sites are located.

placental barrier, and the capacity for firm binding to various cell surfaces are inherent in its structure. All these properties are lost when this portion is removed by proteclytic digestion.

The presence in man and most mammals of several classes of immunoglobulin implies that each class possesses unique properties of survival value. What these are is by no means clear. IgM antibodies are particularly efficient in causing lysis of micro-organisms in the presence of complement, whereas IgG antibodies excel in their ability to react with soluble antigens such as toxins. IgE is particularly endowed with the capacity to fix to mast cells and possibly other tissue components and is the main, if not exclusive, mediator of atopic sensitivity, ${ }^{5}$ though it is difficult at present to associate this with any obvious biological advantage. IgD is virtually an unknown quantity biologically speaking. But IgA has unique properties of great interest. Though its concentration in plasma is only about one-fifth that of $\operatorname{IgG}$, it is the dominant immunoglobulin in the body's secretions such as the tears, saliva, and milk and in the respiratory and alimentary tracts. This ability to pass in considerable quantity through epithelial surfaces is associated with an additional component of some 50,000 molecular weight ${ }^{6}$ which is apparently attached to the molecule during its passage from its plasma cell of origin into the lumen of the secretory gland.

The concentration of IgA-secreting plasma cells in salivary glands and in the respiratory and alimentary tracts, together with the proclivity of this class of immunoglobulin to enter into the various secretions, suggests that it may have a special role in local immunological reactions. This is supported by the observations of Drs. D. B. Kaufman, R. Katz, and R. M. McIntosh at page 463 of the B.M.F. this week. They report that the level of $\operatorname{IgA}$ in the urine of children with chronic or recurrent infections of the urinary tract is greater than normal, while the levels of the other classes of immunoglobulins are not significantly different. Why IgA should be of special value in these situations is not clear, especially as it is not complement-fixing. It may perhaps reside in its greater resistance to proteolysis and its bactericidal activity in the presence of lysozyme.

1Tiselius, A., Transactions of the Faraday Society, 1937, 33, 524.

2Porter, R. R., Biochemical fournal, 1959, 73, 119.

${ }^{3}$ Nisonoff, A., Wissler, F. C., and Lipman, L. N., Science, 1960, 132, 1770

"Fleischman, J. B., Porter, R. R., and Press, E. M., Biochemical fournal, 1963, 88, 220 .

'Ishizaka, K., Ishizaka, T., and Hornbrook, M. M., fournal of Immunology,

'Tomasi, T. B., Tan, E. M., Solomon, A., and Prendergast, R. A., Fournal of Experimental Medicine, 1965, 121, 101.

\section{Yet Another Penicillin}

The era of antibiotic discovery has at least for the time being given place to the evolution of new compounds from existing ones by synthesis. The pioneer work on penicillins has been followed by similar studies of cephalosporins, rifamycins, and lincomycin, and most of the new products acclaimed during the past ten years are of this derivation.

They differ from the natural parent substances in two principal ways. Firstly, in some of them the antibacterial activity is either enhanced, as in ampicillin or carbenicillin against some enterobacteria, or actually. different, as in the resistance of methicillin or cloxacillin to staphylococcal penicillinase. The second property in which improvement has been achieved is absorbability from the alimentary tract. Cephalexin is the only derivative of cephalosporin $C$ which is adequately absorbed after oral administration, and rifampicin the only such derivative of rifamycin. Clindamycin is also better absorbed than its parent, lincomycin. It has recently been reported ${ }^{1}$ that an ester of ampicillin is much better absorbed than ampicillin itself, attaining peak blood concentrations about three times higher after administration of equivalent doses whether in the fasting state or after a meal.

Better absorption is one of the advantages of a new isoxazole penicillin described in the B.M.F. this week (page 455) by Mr. R. Sutherland, Dr. E. A. P. Croydon, and Dr. G. N. Rolinson, of the Beecham Research Laboratories. This group of penicillins shares with methicillin the outstanding property of full activity against staphylococci resistant to benzylpenicillin. They have two further advantages over methicillin, in acid stability, permitting oral administration, and of generally higher antibacterial activity, the difference in their favour against staphylococci being about eight-fold. To set against these is the single drawback of a far higher degree of protein binding, indeed much the highest among penicillins, whereas that of methicillin is among the lowest. If it is accepted that protein-bound antibiotic is antibacterially inactive, then the proportion of free methicillin is so much higher as to compensate fully for its lesser intrinsic activity. This at least is another factor which should be reckoned with in assessing merit.

It may seem strange that after the discovery of this group of compounds in 1961 cloxacillin came into clinical use in Great Britain while the U.S.A. adopted oxacillin. Such differences as there are between them, including stability and degree of antistaphylococcal activity, appear to favour cloxacillin, and American authors ${ }^{2}$ have recognized this. The main difference is in absorption, cloxacillin attaining blood levels twice as high as those of oxacillin. The situation was then altered in the U.S.A. by the introduction of dicloxacillin, containing two chlorine atoms instead of one, which in its turn attains blood levels about twice those of cloxacillin. On the other hand, it is even more highly protein-bound, and the view has been taken that this property cancels the advantage of better absorption.

This is the background against which to consider the new Beecham compound, flucloxacillin, in which one of the chlorine atoms in what would otherwise be dicloxacillin has been replaced by fluorine. The properties of this substance have been thoroughly examined and compared with those of its three relatives. In antibacterial activity against numerous species there is almost nothing to choose between them, except

\footnotetext{
1 Daehne, W. V., et al., fournal of Medicinal Chemistry, 1970, 13, 607. Sidell, S., Bulger, R. J., Brodie, J. L., and Kirby, W. M. M., Clinical Pharmacology and Therapeutics, 1964, 5, 26.
} 